\title{
Anti-amyloidogenic Activity of IgGs Contained in Normal Plasma
}

\author{
Brian O'Nuallain • Angela D. Williams • Helen P. McWilliams-Koeppen • Luis Acero • \\ Alfred Weber • Hartmut Ehrlich • Hans P. Schwarz • Alan Solomon
}

Published online: 20 April 2010

(C) Springer Science+Business Media, LLC 2010

\begin{abstract}
Introduction We have previously shown that a subpopulation of naturally occurring human IgGs has therapeutic potential for the amyloid-associated disorders. These molecules cross-react with conformational epitopes on amyloidogenic assemblies, including amyloid beta $(A \beta)$ protein fibrils that are a pathological hallmark of Alzheimer's disease.

Materials and Methods Using our europium-linked immunosorbant assay, we established that $~ 95 \%$ of 260 screened donor plasma samples had amyloid fibril-reactive IgGs and $A \beta$ conformer-reactive IgGs with minimal binding to $A \beta$ monomers. Anti-amyloidogenic reactivity was diverse and attributed to $A \beta$ targeting multiple fibril-related binding sites and/or variations in multidentate binding.

Results and Discussion There was no correlation between anti-fibril and anti-oligomer reactivity and donor age (19 to 60 years old) or gender. These findings demonstrate the inherent but diverse anti-amyloidogenic activity of natural IgGs contained in normal plasma.

Conclusion Our studies provide support for investigating
\end{abstract}

B. O'Nuallain · A. D. Williams · H. P. McWilliams-Koeppen •

L. Acero $\cdot$ A. Solomon

Human Immunology and Cancer Program,

Department of Medicine,

University of Tennessee Graduate School of Medicine,

Knoxville, TN 37920, USA

A. Weber $\cdot$ H. Ehrlich $\cdot$ H. P. Schwarz

Baxter Bioscience,

Vienna, Austria

B. O’Nuallain $(\bowtie)$

The Conway Institute, University College Dublin,

Belfield,

Dublin 4, Ireland

e-mail: brian.onuallain@ucd.ie the clinical significance and physiological function of this novel class of antibodies.

Keywords Natural $\cdot$ IgGs $\cdot$ amyloid $\cdot A \beta \cdot I V I G$

\section{Introduction}

A pathological hallmark of Alzheimer's disease (AD) and more than 25 other incurable protein misfolding diseases, called the amyloidoses, is the extracellular deposition of amyloid fibrils from unrelated proteins or peptides [1]. The primary culprits for $\mathrm{AD}$ are prefibrillar amyloidogenic assemblies of amyloid beta $(A \beta)$, a proteolyzed transmembrane 39-43 fragment of amyloid precursor protein [2-4]. However, fibril deposition may be the primary toxic event for several non-AD amyloidoses, including primary AL amyloidosis [5].

Currently, immunotherapy directed against amyloidogenic protein is the primary therapeutic approach for $\mathrm{AD}$ and is a recent strategy for other amyloid-associated disorders [6]. Active vaccination with $A \beta$ or passive administration of anti-A $\beta$ antibodies has shown promise in $\mathrm{AD}$ animal models and in some $\mathrm{AD}$ patients by inducing neuritic plaque clearance, neutralizing neurotoxic $A \beta$ oligomers, and/or improving cognitive functioning [7-9]. Antibodies that specifically react with pathogenic amyloidogenic aggregates that do not bind to the normally secreted precursor protein are less likely to have adverse effects. Presumably, such natural IgG antibodies contained in intravenous immunoglobulin (IVIG) are at least partly responsible for its promising clinical effects $[10,11]$. We have recently shown that these antibodies cross-react with conformational epitopes on amyloid fibrils and oligomers, including binding to cross-linked $\beta$-amyloid protein species (CAPS) [12]. These antibodies also inhibit in vitro amyloid fibril growth 
and expedite the clearance of patient-derived amyloid in mice $[12,13]$. The results of our studies on a large number of plasma donor specimens provide new information on the extent and specificity of natural anti-amyloidogenic conformer-reactive IgGs, which are contained in presumably healthy individuals.

\section{Methods}

Peptides, Proteins, and Antibodies Human 40- and 42-mer $A \beta$ peptides were purchased from Quality Controlled Biochemicals (Hopkinton, MA, USA); mass spectrometric analysis showed them to be $>90 \%$ pure. Before using, the lyophilized $A \beta 40$ peptide was disaggregated by sequential exposure to trifluoroacetic acid, hexafluoroisopropanol (HFIP; Pierce, Rockford, IL, USA), and $2 \mathrm{mM} \mathrm{NaOH}$, followed by $2 \times$ PBS $(1 \times$ final). The ultracentrifuged sample yielded a final peptide concentration of $\sim 0.2 \mathrm{mg} / \mathrm{mL}$ [12]. The soluble 42-mer peptide was prepared at $\sim 0.04 \mathrm{mg} / \mathrm{mL}$ in PBS by pretreatment with HFIP/NaOH [12]. The peptide concentrations were determined at $A_{215} \mathrm{~nm}$ by reverse-phase HPLC using an $A \beta 40$ standard curve or by the MicroBCA assay (Pierce).

Recombinant immunoglobulin light chain (LC) $\lambda 6$ variable domain, Jto, was produced in an Escherichia coli expression system and purified using Amberlite XAD-7 (Sigma-Aldrich, St. Louis, MO, USA) [13]. The soluble LC was sterile-filtered using a $0.22-\mu \mathrm{m}$ polyvinylidene fluoride 25-mm Millex-GV syringe-driven filter unit (Millipore, Bedford, MA, USA). SDS-PAGE analyses confirmed that the protein was $>90 \%$ pure, and protein concentration was determined by the MicroBCA assay.

Normal donor plasma, coagulation reference plasma, which consisted of pooled plasma from healthy donors aged 20 to 60, and IVIG (Gammagard liquid) were provided by Baxter BioScience (Vienna, Austria). The blocking agent, essentially fatty-acid-free bovine serum albumin, was purchased from Sigma. All other reagents were of analytical grade.

Preparation of Peptide and Protein Aggregates Soluble CAPS was prepared from the synthetic 40 - or 42 -mer A $\beta$ peptides by incubation with $1.1 \mu \mathrm{M}$ horseradish peroxidase and $250 \mu \mathrm{M} \mathrm{H}_{2} \mathrm{O}_{2}$ in PBS at $37^{\circ} \mathrm{C}$ for $3 \mathrm{~h}$, and then purified using copper $\left(\mathrm{CuSO}_{4}\right)$ precipitation [12]. CAPS was quantified using SDS PAGE (4-12\% Bis Tris precast gels; Invitrogen, Carlsbad, CA, USA) and the MicroBCA assay. Electrospray ionization mass spectrometry (Applied Biosystems, Foster City, CA, USA) and dityrosine fluorescence (excitation at $320 \mathrm{~nm}$ and emission between 350 and $550 \mathrm{~nm}$ ) confirmed that the aggregates consisted of low molecular weight $(<38 \mathrm{kDa})$, cross-linked SDS stable species.
A $\beta 40$ and LC fibrils were grown from the soluble precursor proteins in PBS containing $0.02 \%$ sodium azide (PBSA). The reaction was monitored by thioflavin $\mathrm{T}$ fluorescence [13]. Fibrils were harvested by centrifugation at $20,200 \times g$ for $30 \mathrm{~min}$ at room temperature, then sonicated $(2 \times 30$-s bursts) with a probe sonicator disruptor (Teledyne Tekmar, Mason, $\mathrm{OH}, \mathrm{USA}$ ), aliquoted, and stored at $-20^{\circ} \mathrm{C}$.

$\operatorname{IgG}$ Purification IgGs from donor plasma pools were isolated using a Melon Gel IgG Spin Purification Kit (Pierce). SDS-PAGE confirmed that the resultant samples were $>95 \%$ pure. Antibody concentration was determined by absorbance at $\mathrm{A}_{280 \mathrm{~nm}}$ with the use of a molar extinction coefficient of $210,000 \mathrm{M}^{-1} \mathrm{~cm}^{-1}$.

Antibody Binding Assay Antibody reactivity with amyloid fibrils, CAPS, and monomeric A $\beta 40$ was determined at $37^{\circ}$ $\mathrm{C}$ using the plate-immobilized conformers in our europiumlinked immunosorbant assay (EuLISA) [13]. All measurements were done in triplicate (error bars in the figures represent $\mathrm{SD}$ ), and antibody concentrations that gave halfmaximal binding $\left(\mathrm{EC}_{50}\right.$ values) were determined from the sigmoidally fit binding curves (SigmaPlot 2000 ver. 6; Systat Software, Chicago, IL, USA).

The EuLISA was performed with a 1:20 or serial dilution of plasma or purified IgGs in assay buffer (1\% BSA in PBSA containing $0.05 \%$ Tween 20 ) in activated high-binding microtiter plate wells (COSTAR, Corning, NY, USA) coated with $400 \mathrm{ng}$ of target protein and blocked with $1 \%$ BSA (Sigma-Aldrich) in PBSA. A biotinylated goat anti-human IgG ( $\gamma$-chain specific, Sigma-Aldrich) served as the secondary antibody. After the addition of a $\mathrm{Eu}^{3+}$ streptavidin conjugate, followed by the releasing enhancement solution, $\mathrm{Eu}^{3+}$ time-resolved fluorescence was measured using a Victor $^{2} 1420$ Multilabel Counter (Perkin Elmer, Waltham, MA, USA). The amount (fM) of lanthanide released was calculated from a standard curve using known concentrations of $\mathrm{Eu}^{3+}$.

To establish whether antibody binding was affected by a putative inhibitory plasma molecule, binding studies were done after pretreating the preparation with acid $(0.1 \mathrm{M}$ glycine $\mathrm{HCl}, \mathrm{pH} 3.5$ ), followed by dialysis against PBS (Amicon Ultra-4 filter unit, 30,000 Da m.w. cutoff, from Millipore) to dissociate antigen-antibody complexes [14]. Assay signal was normalized for high-throughput plasma screening using a standard curve on each plate of coagulation reference plasma against the plate-immobilized amyloidogenic conformer.

Assurances Studies involving human specimens were in accordance with a protocol approved by the University of Tennessee Graduate School of Medicine's Institutional Review Board. 


\section{Results}

Anti-amyloidogenic Activity of Natural IgGs Contained in Normal Plasma The construction of full antibody binding curves and a high-throughput screen of 260 plasma established the inherent anti-amyloidogenic conformer reactivity of natural IgGs contained in normal human plasma (Figs. 1 and 2). These molecules were detected in essentially all ( $\sim 95 \%)$ plasma. Surprisingly, the avidity of IgG binding to aggregated amyloidogenic conformers for each sample was similar, with $\mathrm{EC}_{50}$ values of $\sim 100-800 \mathrm{nM}$. The relatively large $\mathrm{EC}_{50}$ values reflected the small fraction, $\sim 0.2 \%$ of polyclonal IgGs in normal plasma that target $A \beta[12,13]$. Diverse maximum binding signal amplitudes were obtained for individual and pooled plasma. These differences were not due to a putative inhibitory plasma molecule, such as endogenous $A \beta$, that interfered with antibody binding [14]. The binding curve for an equimolar mix of high- and lowreactive plasma pools against $\mathrm{A} \beta$ fibrils or CAPS was essentially the same as that for the high-reactive preparations (Figs. 1d and 2d).

Similar with IVIG, natural antibodies contained in normal plasma had comparable reactivity with $\mathrm{A} \beta$ fibrils and CAPS (generated from the 40- and 42-mer $\mathrm{A} \beta$ peptides), but relatively weak interactions with the monomeric peptide (Figs. 1 and 2) [12]. This indicated that natural antiamyloidogenic conformer IgGs display the same amyloid fibril and oligomer cross-reactivity, whether in plasma or as purified molecules [12]. These molecules also bound with
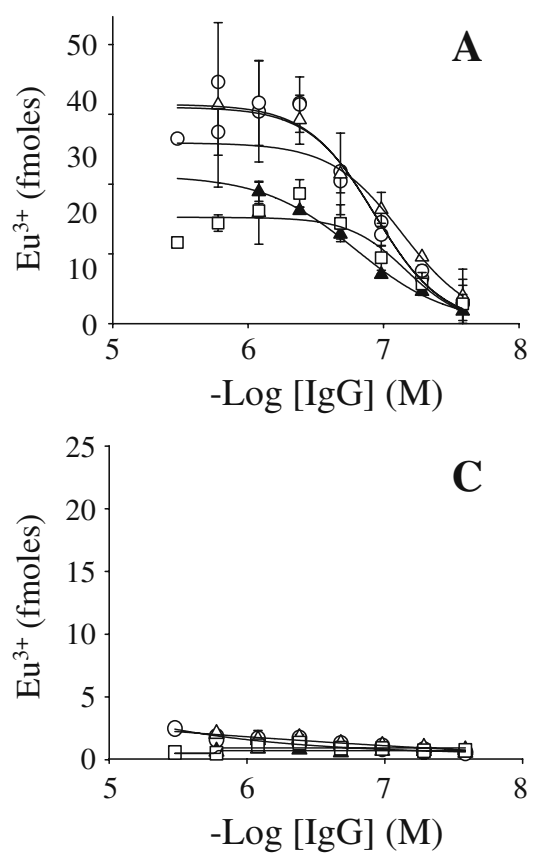

Fig. 1 A $\beta 40$ conformer binding by IgGs contained in normal plasma. Representative antibody binding curves for normal plasma IgGs (filled circle, unfilled circle, unfilled square, filled triangle, unfilled triangle) against $\mathrm{A} \beta$ fibrils (a), CAPS (b), and $\mathrm{A} \beta$ monomers (c). d Binding similarly avidity to $\mathrm{LC}$ fibrils, with $\mathrm{EC}_{50}$ value of $\sim 100$ $300 \mathrm{nM}$ that was consistent with reactivity against conformational epitopes that are less reliant on the amyloidogenic protein's primary sequence (Fig. 2) [13]. Nevertheless, the mean value for plasma $\operatorname{IgG}$ reactivity against $\mathrm{A} \beta$ fibrils, $24 \pm$ $19 \mathrm{Eu}^{3+}$ fmoles, was $\sim 2$-fold greater than that against LC fibrils, $13.4 \pm 13 \mathrm{Eu}^{3+}$ fmoles $(P<0.0001$, paired $t$ test). This indicated that although amyloidogenic conformers have common epitopes, their density can vary.

Our plasma screening results were confirmed by $\sim 5$-fold larger maximum signal amplitude for binding curves for amyloid conformer-reactive IgGs from a pool of 20 plasma that were identified as "high" reactive samples compared with a mixture of "low" binders (Fig. 2b, c). The relatively small signal for the low-reactive plasma pool was not due to a proportion of antibody that was bound to naturally occurring amyloidogenic antigens since similar binding curves were obtained for the purified IgGs, with or without acid pretreatment to dissociate putative antibody-antigen complexes (14; Figs. 2d, e).

Age- and Gender-Related Anti-amyloidogenic Conformer Reactivity There was no significant correlation between antiA $\beta$ fibril and anti-CAPS reactivity with donor age $(\sim 19$ to $\sim 60$ years old; $P=0.238$ and 0.859 for fibril and CAPS reactivity, respectively; nonparametric Kruskal-Wallis test) or gender $(P=0.161$ and 0.058 for fibril and CAPS reactivity, respectively; nonparametric two-tailed Mann-Whitney $t$ test; Fig. 3).
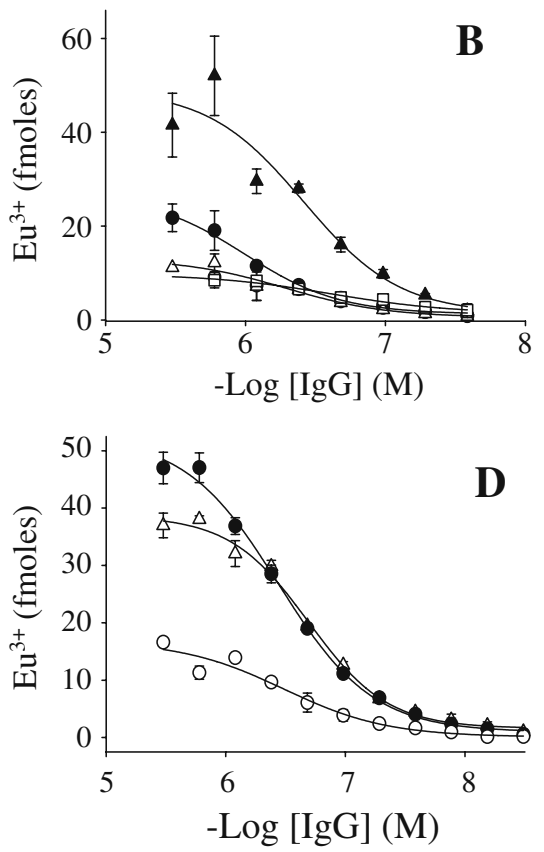

curves for high (filled circle) and low (unfilled circle) reactive plasma pools, and for an equimolar mix (unfilled triangle) against $\mathrm{A} \beta$ fibrils. Each plasma pool consisted of 15 plasma specimens. CAPS indicates dityrosine cross-linked $\beta$-amyloid protein species 

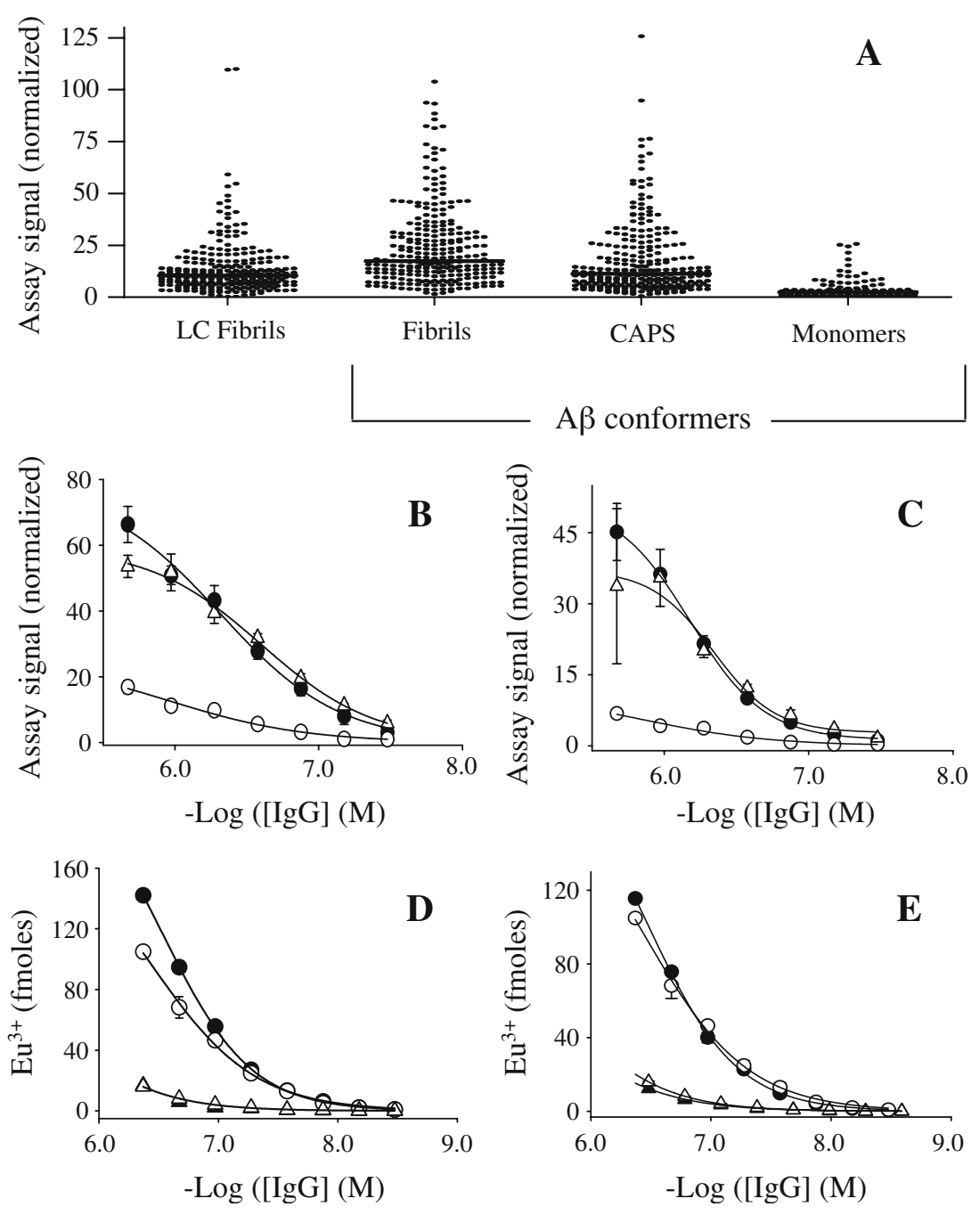

Fig. 2 EuLISA screen of LC fibril and $A \beta$ conformer binding by IgGs contained in 260 donor plasma samples. a Comparison of amyloidogenic conformer IgG binding using a 1:20 dilution of plasma. Solid lines represent the median values, which with the exception of the median value for binding to LC fibrils compared with that for CAPS, significantly differed $(P<0.0001$ in a nonparametric Kruskal-Wallis test). To eliminate plate-to-plate variability, assay signals were normalized using a standard curve constructed from coagulation reference plasma. b-c Antibody binding curves for high (filled circle) and low (unfilled circle) reactive plasma pools and for an equimolar mix (unfilled triangle) against $\mathrm{A} \beta$ fibrils (b), and CAPS generated from the 40 -mer $A \beta$ peptide (c). Similar binding curves to

\section{Discussion}

Given the urgent need for novel therapeutics and diagnostics for amyloid-associated disorders, it is vital that we advance current limited understanding about the prevalence, clinical significance, and physiological function of naturally occurring anti-amyloidogenic conformer IgGs. Our findings demonstrate that these antibodies are inherently present in normal plasma, independent of donor age ( 19-60 years old) or gender. The unique manner in which these IgGs bind to amyloid fibrils and oligomers is evident from their ability to

those shown in (b) and (c) were also determined for the latter plasma pools against LC fibrils and CAPS generated from A $\beta 42$ (data not shown). Each plasma pool consisted of 20 plasma specimens. d Binding curves for purified plasma IgGs from the high (filled circle) and low (filled triangle) reactive plasma pools against LC fibrils compared with curves generated using the respective plasma (unfilled circle, unfilled triangle). e Antibody binding curves for essentially the same reagents described in (d), but each antibody sample was pretreated with acid and dialysis (m.w. cutoff of 30,000 Da), and then neutralized to remove any putative inhibitor of antibodyamyloidogenic conformer interactions. CAPS indicates dityrosine cross-linked $\beta$-amyloid protein species, $L C$ human Ig light chain

maintain reactivity in the presence of endogenous plasma molecules, several of which bind to $A \beta$ [15]. Notably, these antibodies are also present in the plasma of children and naïve mice (B. O'Nuallain, A. Solomon, unpublished data, 2009) [16]. This suggests that they may be part of the innate immune response for clearing endogenous/exogenous "misfolded proteins" by binding to amyloid fibril-like epitopes.

Previous studies have differed on the prevalence and ageand gender-related amyloidogenic conformer reactivity of natural plasma IgGs. [16-18]. These discrepancies presumably reflected the limits of detection by the different assays. 
In some cases, these detection abilities were reduced when screening against disaggregated soluble $A \beta$ with which natural antibodies are only weakly reactive as a result of a surface-induced amyloid-like epitope [12] (Figs. 1 and 2). These discrepancies were unlikely due to screening plasma against different aggregated $A \beta$ conformers, because we have shown that natural IgGs cross-react with common conformational epitopes on all amyloid fibrils or oligomers tested $[12,13]$. Although the current study showed no correlation between the age or gender of plasma donors and anti-amyloidogenic activity, it is possible that an association exists beyond the donor population age range of 19 to
60 years old $[17,18]$. However, screening normal plasma from presumably healthy elderly individuals older than 60 years is problematic because these samples are likely to contain an additional population of amyloid-reactive antibodies that are not naturally occurring but generated against pathologic $A \beta$ assemblies, which may be present decades before $\mathrm{AD}$ is diagnosed.

The molecular basis for natural antibody-amyloidogenic conformer interactions has not been well defined. The epitopes involved are not exposed on native proteins and presumably contain unique higher-ordered structure and/or novel clusters of amino acids. Given that these IgGs react
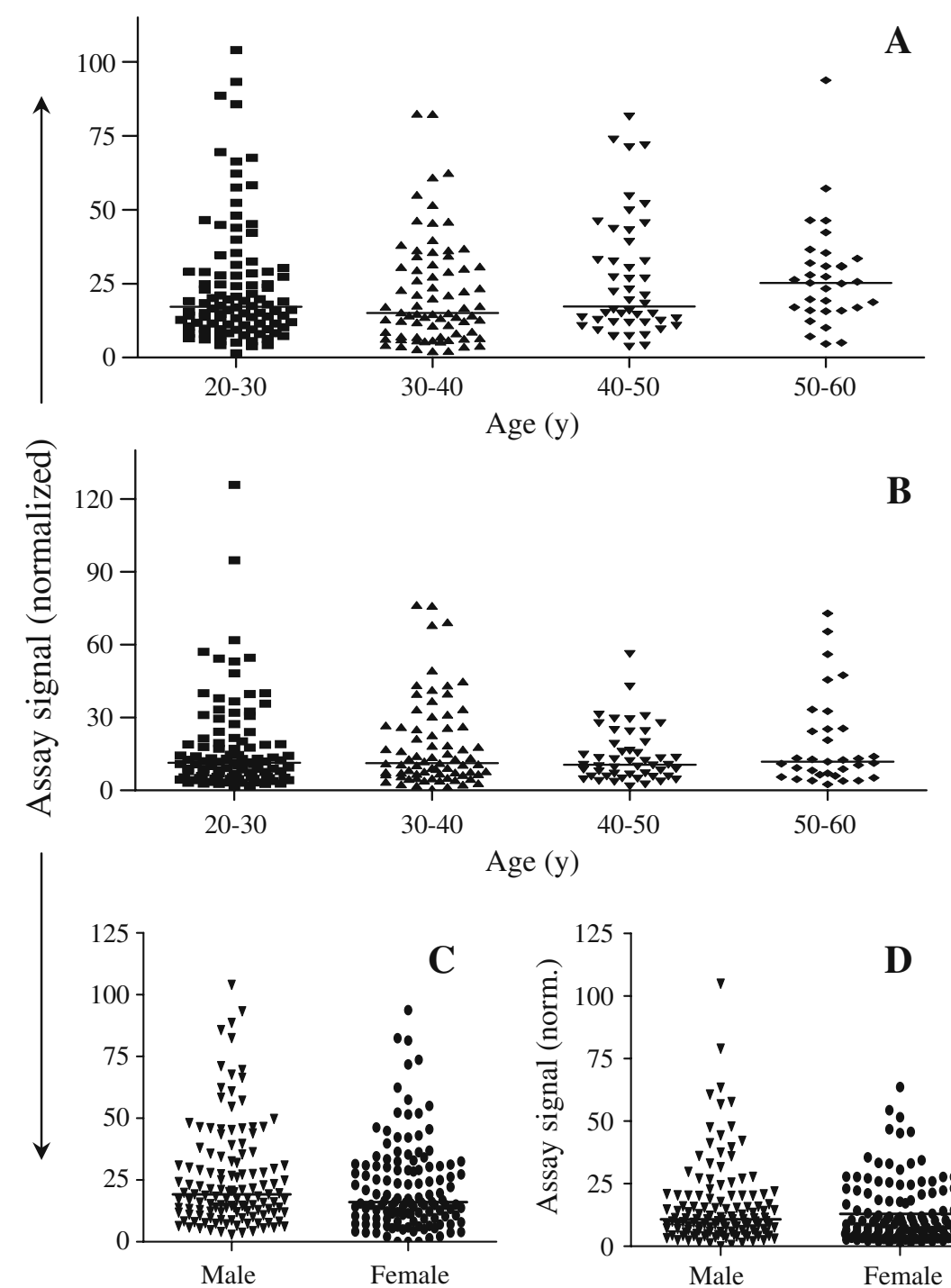

Fig. 3 Donor age- and gender-related anti-amyloidogenic $\operatorname{IgG}$ activity. a-b The plots show donor age versus plasma IgG reactivity against A $\beta 40$ fibrils (a) and CAPS (b). Each solid line represents the median value. The plots show that there was no significant correlation with donor age $(P=0.238$ and 0.859 for fibril and CAPS reactivity, respectively; nonparametric Kruskal-Wallis test). Data were obtained from a bulk screen of 260 normal plasma specimens as shown in Fig. 2. c-d The plots show normal donor gender versus plasma $\operatorname{IgG}$

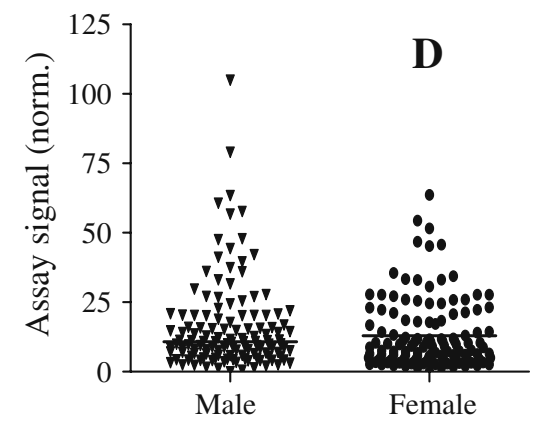

reactivity against $\mathrm{A} \beta$ fibrils (c) and CAPS (d). Each solid line represents the median value. The plots show that there was no significant correlation with donor gender $(P=0.161$ and 0.058 for fibril and CAPS reactivity, respectively; nonparametric two-tailed Mann-Whitney $t$ test). The data were obtained from a screen of 260 normal plasma specimens as shown in Fig. 2. CAPS indicates dityrosine cross-linked $\beta$-amyloid protein species 
with conformational epitopes on "misfolded" protein aggregates, their binding may not involve complementary determining regions that are typically involved in antibodyantigen interactions. Preliminary studies using antibody fragments $\left[F\left(a^{\prime}\right)\right.$ and $\left.F\left(a b^{\prime}\right)_{2}\right]$ indicate that the $F c$ portion and/or hinge region are critical for natural human $\operatorname{IgG}$ binding to aggregated $\mathrm{A} \beta$ (B. O'Nuallain, A. Solomon, unpublished data, 2009). Such interactions may involve a complementary surface that is exposed on a partially unfolded antibody or a superantigen-like binding by the native protein. Nevertheless, antibody reactivity is not due to insoluble antibody aggregates because the same $\operatorname{IgG}$ reactivity with $\mathrm{A} \beta$ fibrils was obtained whether plasma was centrifuged or untreated.

The similar $\mathrm{EC}_{50}$ values that were obtained for high- and low-reactive plasmas are intriguing and indicate that each sample contained a subpopulation of natural IgGs that had comparable avidity for amyloidogenic conformers (Figs. 1 and 2). However, these specimens had diverse maximum binding signal amplitudes, which indicate that each antibody subpopulation differed in its ability to bind to a variety of fibril-related conformational epitopes. Alternatively, this may have reflected the different propensities of the antibody subpopulations for multidentate binding to the same structural repeats.

\section{Conclusions}

We have established that natural IgGs contained in normal plasma produce inherent, but diverse, anti-amyloidogenic activity. Thus, the beneficial effect of IVIG in some AD patients $[10,11]$ may have been due to the enriched diversity of each patient's $A \beta$-reactive IgG pool. This diversity could have improved cognition $[10,11]$ by the enhancement of neuritic plaque clearance $[13,19]$, inhibition of fibril growth $[13,20]$, neutralization of neurotoxic $A \beta$ oligomers [20], and reduction of $A \beta$ brain pool. Taken together, our studies support future investigations of the clinical significance and physiological function of naturally occurring amyloidogenic conformer-reactive antibodies.

Acknowledgments The work of Alan Solomon and Brian O'Nuallain was supported by Baxter BioScience. We thank Daniel Kestler, Jon Wall, and Charles Murphy for their helpful discussions.

\section{References}

1. Stefani M. Protein misfolding and aggregation: new examples in medicine and biology of the dark side of the protein world. Biochim Biophys Acta. 2004;1739:5-25.
2. Haass C, Schlossmacher MG, Hung AY, Vigo-Pelfrey C, Mellon A, Ostaszewski BL, et al. Amyloid beta-peptide is produced by cultured cells during normal metabolism. Nature. 1992;359:322-5.

3. Walsh DM, Selkoe DJ. A beta oligomers - a decade of discovery. J Neurochem. 2007;101:1172-84.

4. Moir RD, Tseitlin KA, Soscia S, Hyman BT, Irizarry MC, Tanzi RE. Autoantibodies to redox-modified oligomeric Abeta are attenuated in the plasma of Alzheimer's disease patients. J Biol Chem. 2005;280:17458-63.

5. Falk RH, Skinner M. The systemic amyloidoses: an overview. Adv Intern Med. 2000;45:107-37.

6. Solomon A, Weiss DT, Wall JS. Immunotherapy in systemic primary (AL) amyloidosis using amyloid-reactive monoclonal antibodies. Cancer Biother Radiopharm. 2003;18:853-60.

7. Gilman S, Koller M, Black RS, Jenkins L, Griffith SG, Fox NC, et al. Clinical effects of Abeta immunization (AN1792) in patients with AD in an interrupted trial. Neurology. 2005;64:1553-62.

8. Klyubin I, Betts V, Welzel AT, Blennow K, Zetterberg H, Wallin A, et al. Amyloid beta protein dimer-containing human CSF disrupts synaptic plasticity: prevention by systemic passive immunization. J Neurosci. 2008;28:4231-7.

9. Solomon B. Alzheimer's disease and immunotherapy. Curr Alzheimer Res. 2004;1:149-63.

10. Dodel RC, Du Y, Depboylu C, Hampel H, Frölich L, Haag A, et al. Intravenous immunoglobulins containing antibodies against beta-amyloid for the treatment of Alzheimer's disease. J Neurol Neurosurg Psychiatry. 2004;75:1472-4.

11. Relkin NR, Szabo P, Adamiak B, Burgut T, Monthe C, Lent RW, et al. 18-Month study of intravenous immunoglobulin for treatment of mild Alzheimer disease. Neurobiol Aging. 2009;30:1728-36.

12. .O'Nuallain B, Acero L, Williams AD, Koeppen HP, Weber A, Schwarz HP, et al. Human plasma contains cross-reactive Abeta conformer-specific IgG antibodies. Biochemistry. 2008;47:12254-6.

13. O'Nuallain B, Hrncic R, Wall JS, Weiss DT, Solomon A. Diagnostic and therapeutic potential of amyloid-reactive IgG antibodies contained in human sera. J Immunol. 2006;176:70718.

14. Li Q, Gordon M, Cao C, Ugen KE, Morgan D. Improvement of a low $\mathrm{pH}$ antigen-antibody dissociation procedure for ELISA measurement of circulating anti-Abeta antibodies. BMC Neurosci. 2007;8:22.

15. Kuo YM, Emmerling MR, Lampert HC, Hempelman SR, Kokjohn TA, Woods AS, et al. High levels of circulating Abeta42 are sequestered by plasma proteins in Alzheimer's disease. Biochem Biophys Res Commun. 1999;257:787-91.

16. Baril L, Nicolas L, Croisile B, Crozier P, Hessler C, Sassolas A, et al. Immune response to Abeta-peptides in peripheral blood from patients with Alzheimer's disease and control subjects. Neurosci Lett. 2004;355:226-30.

17. Mruthinti S, Buccafusco JJ, Hill WD, Waller JL, Jackson TW, Zamrini EY, et al. Autoimmunity in Alzheimer's disease: increased levels of circulating IgGs binding Abeta and RAGE peptides. Neurobiol Aging. 2004;25:1023-32.

18. Xu W, Kawarabayashi T, Matsubara E, Deguchi K, Murakami T, Harigaya Y, et al. Plasma antibodies to Abeta40 and Abeta42 in patients with Alzheimer's disease and normal controls. Brain Res. 2008;1219:169-79.

19. Istrin G, Bosis E, Solomon B. Intravenous immunoglobulin enhances the clearance of fibrillar amyloid-beta peptide. J Neurosci Res. 2006;84:434-43.

20. Du Y, Wei X, Dodel R, Sommer N, Hampel H, Gao F, et al. Human anti-beta-amyloid antibodies block beta-amyloid fibril formation and prevent beta-amyloid-induced neurotoxicity. Brain. 2003;126:1935-9. 